\title{
Stable Vector Bundles and Instantons ${ }^{\star}$
}

\author{
Robin Hartshorne ${ }^{\star \star}$ \\ Department of Mathematics, University of California, Berkeley, California 94720, USA
}

\begin{abstract}
Methods of abstract algebraic geometry are used to study rank 2 stable vector bundles on $\mathbb{P}^{3}$. These bundles are then used to give self-dual solutions, called instantons, of the Yang-Mills equation on $S^{4}$.
\end{abstract}

\section{§0. Introduction}

A problem which has recently attracted considerable attention in the gauge theories of elementary particle physics is the search for self-dual solutions of the Yang-Mills equation. The solutions found so far depend on a finite set of points of the 4-sphere $S^{4}$, and are called instantons. In the language of differential geometry, this problem can be phrased as follows : find all possible connections with self-dual curvature on a smooth SU(2)-bundle over the 4-sphere $S^{4}$. Following the Penrose program of translating physical problems into problems of several complex variables, Atiyah and Ward [2] have shown that this problem is equivalent to the classification of certain holomorphic $\mathbb{C}^{2}$-bundles on complex projective 3 -space $\mathbb{P}_{\mathbb{C}}^{3}$ with an added reality condition (described explicitly in $\$ 1$ ).

An instanton has a "topological quantum number" $k$, which is a positive integer. The physicists [7] have already shown the existence of instantons for all values of $k=1,2, \ldots$. In the differential-geometric interpretation, $k$ is the second Chern class $c_{2}$ of the $\mathrm{SU}(2)$-bundle; it is known that $c_{2}$ completely determines a smooth SU(2)-bundle on $S^{4}$ up to isomorphism. Atiyah et al. [1] have shown that the moduli (or parameter space) for the set of connections with self-dual curvature on the $\mathrm{SU}(2)$-bundle with given $c_{2}>0$ is a smooth manifold of dimension $8 c_{2}-3$. Their proof, which uses deformation theory and the index theorem, is purely local and tells nothing of the global properties of the moduli space. To find out more about this moduli space, in particular, whether it is connected, and to give an explicit construction for the corresponding instantons, it seems that recent work in

* This article reproduces two lectures I gave in I. M. Singer's seminar on gauge theories, at Berkeley in June 1977. Full details will appear elsewhere [6]

$\star \star$ Partially supported by NSF grant NSF MCS 76-03423, A02 
algebraic geometry on the classification of stable vector bundles on $\mathbb{P}_{\mathbb{C}}^{3}$ should be very useful.

In this paper we will formulate the problem on $\mathbb{P}_{\mathbb{C}}^{3}$, mention some related results in algebraic geometry, and begin the program of studying the moduli space $M(k)$ of instantons with topological quantum number $k$. In particular, we show that $M(1)$ is isomorphic to the open unit ball in $\mathbb{R}^{5}$. Thus it is connected and contractible, as was already noted by Atiyah and Ward. For $k=2$, we show that the moduli space $M(2)$ is connected but not simply connected. At the same time, we find that for $k=1,2$, the instantons already constructed by the physicists [7] are the only possible ones.

The terminology and basic results of algebraic geometry can be found in Hartshorne [5].

\section{§1. Statement of the Problem}

In this section we will state the problem on $\mathbb{P}_{\mathbb{C}}^{3}$ which is equivalent to classifying instantons.

First of all, we need some extra structure, consisting of a map $\pi: \mathbb{P}_{\mathbb{C}}^{3} \rightarrow S^{4}$, and a conjugate linear involution $j: \mathbb{P}_{\mathbb{C}}^{3} \rightarrow \mathbb{P}_{\mathbb{C}}^{3}$ which preserves the fibres of $\pi$. To define these, let $\mathbb{H}$ be the ring of quaternions, that is, a 4-dimensional real vector space with basis $1, i, j, k$, and multiplication determined by $i^{2}=j^{2}=k^{2}=-1, i j=k, j k=i$, $k i=j, j i=-k, k j=-i, i k=-j$. We identify a four-dimensional complex vector space $\mathbb{C}^{4}$, having coordinates $z_{0}, z_{1}, z_{2}, z_{3}$, with a two-dimensional quaternion space $\mathbb{H}^{2}$, having coordinates $w_{0}, w_{1}$, by setting

$$
\begin{aligned}
& w_{0}=z_{0}+z_{1} j \\
& w_{1}=z_{2}+z_{3} j .
\end{aligned}
$$

Now let $j$ denote left multiplication on $\mathbb{H}^{2}$ by $j$. This induces a map $j: \mathbb{C}^{4} \rightarrow \mathbb{C}^{4}$ which is easy to make explicit:

$$
j w_{0}=j z_{0}+j z_{1} j=\bar{z}_{0} j+\bar{z}_{1} j^{2}=-\bar{z}_{1}+\bar{z}_{0} j,
$$

and similarly for $w_{1}$. Here we use the fact that for any complex number $z=a+b i$, considered as a quaternion, $j z=\bar{z} j$. Thus $j$ on $\mathbb{C}^{4}$ is the map

$$
j\left(z_{0}, z_{1}, z_{2}, z_{3}\right)=\left(-\bar{z}_{1}, \bar{z}_{0},-\bar{z}_{3}, \bar{z}_{2}\right) .
$$

This map is conjugate linear in the sense that $j(\lambda v)=\bar{\lambda} j(v)$ for any $\lambda \in \mathbb{C}, v \in \mathbb{C}^{4}$. Note also that $j^{2}$ is just multiplication by -1 on $\mathbb{C}^{4}$.

Next we define the map $j: \mathbb{P}_{\mathbb{C}}^{3} \rightarrow \mathbb{P}_{\mathbb{C}}^{3}$ by letting $j$ act on the homogeneous coordinates $z_{0}, z_{1}, z_{2}, z_{3}$ as above. This makes sense, because if we multiply the coordinates of a point $P$ by $\lambda$, then the coordinates of $j(P)$ are multiplied by $\bar{\lambda}$, so they still represent the same point. Since $j^{2}$ multiplies the coordinates by -1 , it is the identity map on $\mathbb{P}_{\mathbb{C}}^{3}$, i.e. $j$ is an involution.

Now let $\mathbb{P}_{\mathbb{H}}^{1}$ be the projective line over $\mathbb{H}$, defined as equivalence classes of ordered pairs $\left(w_{0}, w_{1}\right), w_{i} \in \mathbb{H}$, under the equivalence relation $\left(w_{0}, w_{1}\right) \sim\left(\alpha w_{0}, \alpha w_{1}\right)$ for any $\alpha \in \mathbb{H}$. This space $\mathbb{P}_{\mathbb{H}}^{1}$ is topologically a 4-sphere $S^{4}$. The natural projection map $\mathbb{H}^{2}-\{0\} \rightarrow \mathbb{P}_{\mathbb{H}}^{1}$ induces a map $\pi: \mathbb{P}_{\mathbb{C}}^{3} \rightarrow \mathbb{P}_{\mathbb{H}}^{1}=S^{4}$ via the identification of $\mathbb{H}^{2}$ with $\mathbb{C}^{4}$ above. Note that by construction the fibres are stable under the map $j$. 
Note finally that a fibre of $\pi$ is the image in $\mathbb{P}_{\mathbb{C}}^{3}$ of a 1-dimensional subspace of $\mathbb{H}^{2}$, namely an $\mathbb{H} \cong \mathbb{C}^{2}$, which gives a $\mathbb{P}_{\mathbb{C}}^{1}$ in $\mathbb{P}_{\mathbb{C}}^{3}$. This is topologically a 2-sphere $S^{2}$. Under this identification, one can show that $j$ is essentially the antipodal map on each fibre.

Now we can state our problem.

Problem 1.1. Classify all holomorphic rank 2 vector bundles $E$ on $\mathbb{P}_{\mathbb{C}}^{3}$, together with a map $\tilde{j}: E \rightarrow E$, such that

(1) $c_{1}(E)=0, c_{2}(E)>0$, where $c_{1}$ and $c_{2}$ are the Chern classes, which we consider as integers;

(2) $E$ is stable (which in the presence of (1) is equivalent to saying $H^{0}(E)=0:$ E has no global holomorphic sections);

(3) for each point $p \in S^{4},\left.E\right|_{\pi^{-1}(p)}$ is the trivial rank 2 bundle on $\pi^{-1}(p)=\mathbb{P}_{\mathbb{C}}^{1}$;

(4) the map $\tilde{j}: E \rightarrow E$ is a conjugate linear map, lying over the involution $j: \mathbb{P}_{\mathbb{C}}^{3} \rightarrow \mathbb{P}_{\mathbb{C}}^{3}$, such that $\tilde{j}^{2}=-\mathrm{id}_{E}$.

Furthermore, we should consider E up to isomorphism of holomorphic bundles, and $\tilde{j}$ up to multiplication by $\lambda \in \mathbb{C},|\lambda|=1$. (Note that if $|\lambda|=1$, then $(\lambda \tilde{j})^{2}=\lambda \tilde{j} \lambda \tilde{j}$ $=\lambda \tilde{\lambda} \tilde{j}^{2}=\tilde{j}^{2}$.)

This is the problem which Atiyah and Ward [2] have shown is equivalent to the problem of classifying instantons.

The problem naturally falls into two parts. The first part is to classify all holomorphic rank 2 vector bundles on $\mathbb{P}_{\mathbb{C}}^{3}$ satisfying (1) and (2). This is a purely holomorphic question over $\mathbb{C}$, which does not involve the additional structure $j$. Note that in contrast to the differential-geometric version, where there is just one $\mathrm{SU}(2)$-bundle on $\mathrm{S}^{4}$ with given Chern class $c_{2}$, and the problem is to classify certain connections on the given bundle, here there are many different holomorphic bundles with the given Chern classes. By virtue of a theorem of Serre [9] this holomorphic problem is equivalent to the algebro-geometric problem of classifying algebraic rank 2 vector bundles on the algebraic variety $\mathbb{P}_{\mathbb{C}}^{3}$, subject to the same two conditions (1) and (2). We will discuss this algebro-geometric problem in $\S 2$.

The second part of the problem is to determine which bundles satisfying (1) and (2) also satisfy (3), and admit a map $\tilde{j}$ satisfying (4). We will see later that such a $\tilde{j}$, if it exists on a given bundle $E$, is unique. Therefore the set of solutions of the whole problem will be a subset of the set of solutions to the first part of the problem. This second part also can be phrased in algebraic geometry, this time algebraic geometry over the real numbers. We will discuss this in $\S 3$.

\section{§2. Stable Vector Bundles on $\mathbb{P}_{\mathbb{C}}^{3}$}

Let us consider algebraic rank 2 vector bundles on $\mathbb{P}_{\mathbb{C}}^{3}$. For any such bundle $E$, let $c_{1}(E)$ and $c_{2}(E)$ be its Chern classes, considered as integers. It is known, for purely topological reasons, that $c_{1} c_{2} \equiv 0(\bmod 2)$. Furthermore, it is known that for any pair of integers $c_{1}, c_{2}$ satisfying $c_{1} c_{2} \equiv 0(\bmod 2)$, there is an algebraic rank 2 vector bundle on $\mathbb{P}_{\mathbb{C}}^{3}$ with those Chern classes. In fact, one can show that for any such given $c_{1}$ and $c_{2}$, there are algebraic families of arbitrarily large dimension of mutually nonisomorphic bundles with those Chern classes. This may make the classification problem seem hopeless. However, if one imposes the additional 
restriction that the bundles should be stable - a restriction first suggested by Mumford, and later used by many others - the problem becomes manageable. In this particular context, Maruyama [8] has shown that the family of all stable rank 2 bundles on $\mathbb{P}_{\mathbb{C}}^{3}$ with given Chern classes $c_{1}$ and $c_{2}$ can be parametrized by a finite union of quasi-projective algebraic varieties. Fortunately for us, the bundles which occur in Problem 1.1 are all stable, so we can ignore the others. Furthermore, we need only consider bundles with $c_{1}=0$, in which case the stability condition implies $c_{2}>0$.

Our principal tool for studying these bundles is to associate a bundle with an algebraic curve in $\mathbb{P}_{\mathbb{C}}^{3}$. This is done as follows. Let $\mathcal{O}(1)$ denote the line bundle on $\mathbb{P}_{\mathbb{C}}^{3}$ corresponding to a hyperplane. For any bundle $E$, we denote by $E(n)$ the twisted bundle $E \otimes \mathcal{O}(1)^{\otimes n}$. A theorem of Serre asserts that for $n$ sufficiently large, $E(n)$ will be generated by global sections. In particular it has non-zero global sections, even though $E$ itself does not. Let $s \in H^{0}(E(n))$ be a non-zero global section, and let $(s)_{0}=Y \subseteq \mathbb{P}_{\mathbb{C}}^{3}$ be the zero-set of that section. Then (provided $s$ was sufficiently general) $Y$ will be an algebraic curve in $\mathbb{P}_{\mathbb{C}}^{3}$. In this way we associate to $E$ a curve $Y$, depending of course on $n$ and $s$. If we assume that $c_{1}(E)=0$ and let $c_{2}(E)=c_{2}$, then one can compute the degree $d$ and the genus $g$ of $Y$ as follows: $d=c_{2}+n^{2} ; g=d(n-2)+1$. Furthermore, the canonical sheaf $\omega_{Y}$ on $Y$ is isomorphic to $\mathcal{O}_{Y}(2 n-4)$. In particular, it is the restriction of a line bundle on $\mathbb{P}_{\mathbb{C}}^{3}$.

The real importance of this construction is that it has a converse. We can say exactly which curves arise from vector bundles, and give an existence theorem for a bundle associated to a given curve. The precise result, following an idea due originally to Serre, is this.

Theorem 2.1. There is a one-to-one correspondence between (a) the set of pairs $\langle E, s\rangle$, where $E$ is a rank 2 vector bundle on $\mathbb{P}_{\mathbb{C}}^{3}$ with $c_{1}(E)=0$, and $s \in H^{0}(E(n))$ is a global section for some $n$, and (b) the set of curves $Y$ in $\mathbb{P}^{3}$, together with a given isomorphism $\omega_{Y} \cong \mathcal{O}_{Y}(2 n-4)$. Furthermore, the bundle $E$ is stable if and only if the curve $Y$ is not contained in any surface of degree $\leqq n$.

Technical Note. To make this exact, in (a) one must require that the zero set $(s)_{0}$ of $s$ have codimension 2 in $\mathbb{P}_{\mathbb{C}}^{3}$, and in (b) one should allow the word "curve" to mean a locally complete intersection closed subscheme of $\mathbb{P}_{\mathbb{C}}^{3}$, possibly reducible and possibly with nilpotent elements. A theorem of Kleiman implies that for $n$ sufficiently large, and for $s$ sufficiently general, the curve $Y$ will be irreducible and nonsingular; however, it is often preferable to use a smaller value of $n$, even at the expense of having nilpotent elements in $Y$.

Proof. To go from (a) to (b), we simply take $Y=(s)_{0}$. To go from (b) to (a), we construct the sheaf $E(n)$ as an extension

$$
0 \rightarrow \mathcal{O}_{\mathbb{P}^{3}} \rightarrow E(n) \rightarrow \mathscr{I}_{Y}(2 n) \rightarrow 0,
$$

where $\mathscr{I}_{Y}$ is the ideal sheaf of the curve $Y$. To give such an extension it is equivalent to give an element $\xi \in \operatorname{Ext}_{\mathfrak{O}_{\mathbb{P} 3}}^{1}\left(\mathscr{I}_{Y}(2 n), \mathcal{O}_{\mathbb{P}^{3}}\right)$. Using various exact sequences and canonical identifications, one can show that this Ext group is isomorphic to $H^{0}\left(Y, \omega_{Y}(4-2 n)\right)$. Thus $\xi$ is determined by giving the isomorphism of $\omega_{Y}$ with $\mathcal{O}_{Y}(2 n-4)$. 
This theorem is valuable in that it gives an existence theorem for bundles associated to certain curves. It is not so useful for constructing these bundles explicitly (e.g., giving their transition functions), because it is difficult to chase through the exact sequences and the Ext construction used in the proof. However, it is fine if we are only interested in the moduli space of all such bundles, because we can relate that to the moduli space of curves, which is easier to handle. Of course the curve $Y$ depends on the section $s$ as well as the bundle $E$, so to get the moduli space for bundles $E$, we will have to eliminate the ambiguity introduced by $s$.

Example 2.2. Let $Y$ be a disjoint union of $r$ lines $\mathbb{P}_{\mathbb{C}}^{1}$ in $\mathbb{P}_{\mathbb{C}}^{3}$, with $r \geqq 2$. The canonical sheaf of a line is $\mathcal{O}(-2)$. Thus $\omega_{Y} \cong \mathcal{O}_{Y}(-2)$, and this isomorphism is determined up to $r$ non-zero complex numbers $\lambda_{1}, \ldots, \lambda_{r} \in \mathbb{C}^{*}$, one for each component of $Y$. Taking $n=1$, we thus get a bundle $E$ for each choice of $Y$ and each choice of isomorphism $\omega_{Y} \cong \mathcal{O}_{Y}(-2)$. Since $\operatorname{deg} Y=r$, we have $c_{1}(E)=0$ and $c_{2}(E)=r-1$. Furthermore, for $r \geqq 2, Y$ is not contained in a plane, so $E$ will be stable. This shows the existence of stable bundles for all values of $c_{2} \geqq 1$.

Now let us compute the dimension of the family of bundles obtained in this way. To determine a line in $\mathbb{P}_{\mathbb{C}}^{\mathbb{3}}$ requires 4 (complex) parameters. [This is the dimension of the Grassmann variety $G(1,3)$ which parametrizes lines in $\mathbb{P}^{3}$, or equivalently, 2-dimensional subvector spaces of a 4-dimensional vector space $\mathbb{C}^{4}$.] Thus the choice of $Y$ requires $4 r$ parameters. The choice of the isomorphism $\omega_{Y} \cong \mathcal{O}_{Y}(-2)$ depends on the $r$ parameters $\lambda_{1}, \ldots, \lambda_{r}$. Thus the pair $\left\langle E, s \in H^{0}(E(1))\right\rangle$ depends on $5 r$ parameters. Now we should subtract $\operatorname{dim} H^{0}(E(1))$, which is the number of parameters in the choice of $s$. One can show that $\operatorname{dim} H^{0}(E(1))=5$ if $r=2 ; 2$ if $r=3 ; 1$ if $r \geqq 4$. Thus we see that the bundles constructed by this method form an (irreducible) algebraic family of dimension

$$
\begin{array}{rll}
5 & \text { if } & c_{2}=1 \\
13 & \text { if } & c_{2}=2 \\
5 c_{2}+4 & \text { if } & c_{2} \geqq 3 .
\end{array}
$$

This example is closely related to the specific instantons constructed by the physicists. They fix $r$ points in $S^{4}$ (depending on $4 r$ real parameters) and positive real numbers $\lambda_{1}, \ldots, \lambda_{r}>0$, to construct their instantons. Then gauge transformations give some equivalences, so that they end up with families of instantons depending on 5,13 , respectively $5 c_{2}+4$ real parameters. Via the map $\pi: \mathbb{P}_{\mathbb{C}}^{3} \rightarrow S^{4}$, the $r$ points $p_{1}, \ldots, p_{r} \in S^{4}$ correspond to $r$ disjoint lines $\pi^{-1}\left(p_{1}\right), \ldots, \pi^{-1}\left(p_{r}\right) \cong \mathbb{P}_{\mathbb{C}}^{3}$. This shows the connection between the two constructions. Note that the instantons depend upon a number of real parameters that is the same as the number of complex parameters needed to describe the algebraic vector bundles. This is because the lines of the form $\pi^{-1}(p)$ depend on 4 real parameters, and the $\lambda_{i}$ are real in the construction of the instantons.

Example 2.3. Let $Y$ be an irreducible elliptic curve (i.e. Riemann surface of genus 1) in $\mathbb{P}_{\mathbb{C}}^{3}$, of degree $d \geqq 5$. Then $\omega_{Y} \cong \mathcal{O}_{Y}$, and the isomorphism is determined up to a single $\lambda \in \mathbb{C}^{*}$. Taking $n=2$, we therefore obtain a bundle $E$ with $c_{1}=0, c_{2}=d-4$, and a section $s \in H^{0}(E(2))$. For $d \geqq 5, Y$ is not contained in any quadric surface, so $E$ 
will be stable. (For $d=3, Y$ is a plane cubic curve; for $d=4, Y$ is the complete intersection of two quadric surfaces.) Thus again we obtain stable bundles for every value of $c_{2} \geqq 1$. As before, one can compute the dimension of the family of bundles obtained in this way. Without going into details, here is the result: the dimension of the family is

$$
\begin{array}{rll}
5 & \text { if } & c_{2}=1 \\
13 & \text { if } & c_{2}=2 \\
21 & \text { if } & c_{2}=3 \\
29 & \text { if } & c_{2}=4 \\
4 c_{2}+16 & \text { if } & c_{2} \geqq 5 .
\end{array}
$$

For $c_{2}=1,2$, this gives the same answer (and in fact the same bundles) as the previous example. For $c_{2}=3,4$ we get a larger family than in the previous example. These numbers $(21,29)$ are the same as those predicted by the index theorem $\left(8 c_{2}-3\right)$, so we might hope that this construction would give all possible bundles with $c_{2}=3,4$. But for $c_{2} \geqq 5$, the dimensions we get here (and in the previous example) are less than $8 c_{2}-3$, so it is clear that we will need curves of higher genus to construct all possible bundles. Note also that as $c_{2}$ gets large, $4 c_{2}+16<5 c_{2}+4$, so the bundles coming from elliptic curves are not more general than those coming from disjoint lines. They are simply a different family, and presumably both are subfamilies of some larger more general family.

Now an important question arises. Having found a construction for some families of stable bundles, how can we tell whether we have obtained all possible stable bundles with the given Chern classes? Serre's theorem tells us that for any given bundle $E$, some twist $E(n)$ will have global sections, and will therefore correspond to a curve. But as $n$ gets larger, the corresponding curves will have larger degree and genus, therefore will be harder to classify. Because of Maruyama's theorem of the existence of a variety of moduli for stable bundles with given $c_{2}$, and the fact that the twist $n$ required to make $H^{0}(E(n)) \neq 0$ is a semicontinuous function on the parameter space, we can conclude by quasicompactness that for each $c_{2}$, there exists some $n=n\left(c_{2}\right)$, such that for every stable bundle $E$ with $c_{1}=0, c_{2}(E)=c_{2}$, we will have $H^{0}(E(n)) \neq 0$. If we knew this number $n$, then we could in principle find all stable $E$ with the given $c_{2}$, by looking for all curves of degree up to $c_{2}+n^{2}$ whose canonical sheaf $\omega_{Y}$ is isomorphic to a suitable $\mathcal{O}_{Y}(\ell)$. In response to this question we have a conjecture and some partial results.

Conjecture 2.4. Let $E$ be a stable rank 2 bundle on $\mathbb{P}_{\mathbb{C}}^{3}$ with $c_{1}=0$ and $c_{2}>0$. Let $n>\sqrt{3 c_{2}+1}-2$. Then $H^{0}(E(n)) \neq 0$.

Theorem 2.5. The conjecture is true for $c_{2}=1,2, \ldots, 9$. For $c_{2}>9$ the following weaker result holds: if $2 n^{3}+9 n^{2}+13 n+6>3 c_{2}^{2}+3 c_{2}$, then $H^{0}(E(n)) \neq 0$.

The proof will appear in [6].

For example, if $c_{2}=1$, then $H^{0}(E(1)) \neq 0$, so $E$ comes from a curve $Y$ of degree 2 with $\omega_{Y} \cong \mathcal{O}_{Y}(-2)$. Such a curve $Y$ must be either two skew lines, as in (2.2), or a 
scheme structure of multiplicity 2 on a single line. In fact, the latter possibility does not give any bundles not already obtained from two skew lines, so we see that the construction of (2.2) for $r=2$ gives all possible stable bundles with $c_{2}=1$.

For $c_{2}=2$, we again have $H^{0}(E(1)) \neq 0$, so $E$ corresponds to a curve $Y$ of degree 3 , which must be either three skew lines, or a single line plus a double line, or a single line of multiplicity three. Again in this case one can show that all possible stable bundles with $c_{2}=2$ come from three skew lines as in (2.2).

For $c_{2}=3,4$, we find that $H^{0}(E(2)) \neq 0$. This means that any stable bundle with $c_{2}=3$ or 4 must either come from 4 or 5 skew lines, or an elliptic curve of degree 7 or 8 , or a scheme which is a degenerate case of one of these. The analysis of all such special subschemes becomes rather complicated, and has not yet been carried out in these cases.

For $c_{2}=5$, the theorem gives $H^{0}(E(3)) \neq 0$. Thus, as suggested before, we do not expect the construction of (2.3) to give all possible bundles. And indeed, one can show there are stable bundles with $c_{2}=5$ for which $H^{0}(E(2))=0$.

We conclude this section with a few remarks about the moduli space of stable vector bundles. Let $M_{\mathbb{C}}\left(c_{2}\right)$ denote the moduli space of stable rank 2 vector bundles on $\mathbb{P}_{\mathbb{C}}^{3}$ with $c_{1}=0$ and given $c_{2}$. According to Maruyama's theorem, it is a finite union of quasi-projective varieties over $\mathbb{C}$. We can use deformation theory to study the infinitesimal structure in the neighborhood of any given point. If $x \in M_{\mathbb{C}}\left(c_{2}\right)$ is a point corresponding to a bundle $E$, then one knows that the Zariski tangent space to $M$ at $x$ (which is defined as the dual $\mathbb{C}$-vector space to $m_{x} / m_{x}^{2}$ ) has dimension equal to $\operatorname{dim} H^{1}(\mathscr{E} n d(E))$, where $\mathscr{E} n d(E)$ is the sheaf of endomorphisms of $E$. Furthermore, if $H^{2}(\mathscr{E} n d(E))=0$, then $M$ is nonsingular at $x$, so that the dimension of $M$ itself is given by the dimension of the Zariski tangent space, namely $H^{1}(\mathscr{E} n d(E))$.

One knows that a stable vector bundle has no global endomorphisms other than scalar multiplication, so $H^{0}(\mathscr{E} n d(E))=\mathbb{C}$. Therefore we can use the RiemannRoch theorem, applied to $\mathscr{E} n d(E)$ on $\mathbb{P}_{\mathbb{C}}^{3}$ to compute these dimensions. The result is

$$
\operatorname{dim} H^{1}(\mathscr{E} n d(E))-\operatorname{dim} H^{2}(\mathscr{E} n d(E))=8 c_{2}-3 .
$$

If $H^{2}=0$, then $M_{\mathbb{C}}\left(c_{2}\right)$ will be a complex manifold of complex dimension $8 c_{2}-3$, which is the same as the real dimension of the space of instantons $M\left(c_{2}\right)$ given by the index theorem. In some cases, including all the bundles constructed in (2.2) and (2.3), we can prove that $H^{2}(\mathscr{E} n d(E))=0$. However, in contrast to the differentialgeometric situation, where the analogous $H^{2}$ always vanishes, there are examples of stable bundles with $H^{2}(\mathscr{E} n d(E)) \neq 0$.

\section{§3. Real Structures on Complex Varieties}

In this section we will show how conditions (3) and (4) of (1.1) can be reinterpreted in the language of real algebraic geometry. First we will study the general notion of a real structure on a complex variety.

It is well known that the set of solutions of a polynomial equation over the real numbers may not sufficiently reflect properties of the equation itself. For example, the equation $x^{2}+y^{2}=1$ describes a circle in the plane, which is fine. On the other 
hand, the equation $x^{2}+y^{2}=-1$ has no real solutions at all. Surely we do not wish to identify the "algebraic curve over $\mathbb{R}$ defined by $x^{2}+y^{2}=-1$ " (whatever that means) with the empty set. So we need to deal with real algebraic varieties in some way which is more sophisticated than merely looking at the real solutions of polynomial equations. One way of doing this is to use the notion of a scheme over $\mathbb{R}$. Another equivalent way, more suitable for our purposes, is to use the notion of a real structure on a complex variety.

Definition. Let $X$ be an algebraic variety over $\mathbb{C}$ (or scheme over $\mathbb{C}$, or a complex manifold). A real structure on $X$ is a conjugate linear map $\sigma: X \rightarrow X$ with $\sigma^{2}=\mathrm{id}_{X}$. The fixed points of $\sigma$ (if any) are called the real points of $X$ with respect to the real structure $\sigma$.

For example, let $X \subseteq \mathbb{C}^{2}$ be defined by the equation $x^{2}+y^{2}=1$, and let $\sigma$ be complex conjugation. Then $\sigma$ is a real structure on $X$ whose real points form the circle $x^{2}+y^{2}=1$ in $\mathbb{R}^{2}$. On the other hand, if $X$ is defined by $x^{2}+y^{2}=-1$, then $X$ is a curve over $\mathbb{C}$, with a real structure $\sigma$ given by complex conjugation, having no real points.

Definition. Let $X$ be a variety over $\mathbb{C}$ with a real structure $\sigma$. Let $\mathscr{F}$ be a coherent sheaf (or vector bundle) over $X$. A real structure on $\mathscr{F}$ is a conjugate linear map $\tilde{\sigma}: \mathscr{F} \rightarrow \mathscr{F}$ lying over the map $\sigma: X \rightarrow X$, such that $\tilde{\sigma}^{2}=\mathrm{id}_{\mathscr{F}}$. A symplectic structure on $\mathscr{F}$ is the same, except that $\tilde{\sigma}^{2}=-\mathrm{id}_{\mathscr{F}}$. We say two real or symplectic structures $\tilde{\sigma}$ and $\tilde{\sigma}^{\prime}$ are equivalent if $\tilde{\sigma}^{\prime}=\lambda \tilde{\sigma}$ for some $\lambda \in \mathbb{C},|\lambda|=1$.

For example, if $X$ has a real structure $\sigma: X \rightarrow X$, the induced map on the structure sheaf $\tilde{\sigma}: \mathcal{O}_{X} \rightarrow \mathcal{O}_{X}$ will be a real structure on $\mathcal{O}_{X}$. For another example, the map $\tilde{j}$ on $\mathcal{O}_{\mathbb{P}^{3}}(1)$ induced by the real structure $j$ on $\mathbb{P}_{\mathbb{C}}^{3}$ above is a symplectic structure on $\mathcal{O}_{\mathbb{P}^{3}}(1)$. This is because the global sections of $\mathcal{O}(1)$ form the vector space $\mathbb{C}^{4}$ with basis $z_{0}, z_{1}, z_{2}, z_{3}$, where we saw that $j^{2}$ acted as -1 . Finally, the map $\tilde{j}: E \rightarrow E$ postulated in (1.1) is a symplectic structure on $E$.

To illustrate these notions, we will classify the possible real and symplectic structures on a complex vector space. The proofs are elementary (conjugate) linear algebra.

Proposition 3.1. Let $V$ be an $n$-dimensional complex vector space. If $\tilde{\sigma}$ is a real structure on $V$, then there is a basis $e_{1}, \ldots, e_{n}$ of $V$ such that $\sigma\left(e_{i}\right)=e_{i}$ for each $i$. Thus the only real structure on $V$ is the usual one: for any $v=\sum a_{i} e_{i}$ in $V, \tilde{\sigma}(v)=\sum \bar{a}_{i} e_{i}$. If $\tilde{\sigma}$ is a symplectic structure on $V$, then $n$ must be even, say $n=2 r$, and there is a basis $e_{1}, e_{1}^{\prime}, \ldots, e_{r}, e_{r}^{\prime}$ such that $\tilde{\sigma}\left(e_{i}\right)=e_{i}^{\prime}$ and $\tilde{\sigma}\left(e_{i}^{\prime}\right)=-e_{i}$ for each $i$. Equivalently, $V$ has $a$ structure of $r$-dimensional vector space over the quaternions $\mathbb{H}$, with $\tilde{\sigma}$ being multiplication by $j$.

Next we will classify the possible real structures on complex projective space $\mathbb{P}_{\mathbb{C}}^{n}$. The key point is that if $\sigma$ is a real structure on $\mathbb{P}_{\mathbb{C}}^{n}$, then anything which is functorially associated with $\mathbb{P}_{\mathbb{C}}^{n}$ will inherit a real or symplectic structure. Consider for example the sheaf $\mathcal{O}(1)$ associated to a hyperplane. This sheaf can be characterized as the generator of the Picard group of $\mathbb{P}_{\mathbb{C}}^{n}$ (which is isomorphic to $\mathbb{Z}$ ) having global sections [which distinguishes it from $\mathcal{O}(-1)]$. Therefore $\sigma^{*} \mathcal{O}(1)$ must 
be isomorphic to $\mathcal{O}(1)$. An isomorphism $f: \mathcal{O}(1) \rightarrow \sigma^{*} \mathcal{O}(1)$, which is $\mathbb{C}$-linear, can be considered as a conjugate linear map $f: \mathcal{O}(1) \rightarrow \mathcal{O}(1)$ lying over $\sigma$. It is determined up to multiplication by $\lambda \in \mathbb{C}, \lambda \neq 0$. On the other hand, $f^{2}$ is a linear isomorphism of $\mathcal{O}(1)$ with itself, hence $f^{2}=\mu \cdot$ id for some $\mu \in \mathbb{C}$. One sees easily that $\mu$ is real. Then, replacing $f$ by $\lambda f$ for suitable $\lambda, \mu$ is replaced by $|\lambda| \cdot \mu$, so we can arrange that $f^{2}= \pm 1$. Thus $f$ is either a real or a symplectic structure on $\mathcal{O}(1)$, uniquely determined up to equivalence.

Now $f$ induces a real or symplectic structure on the vector space $H^{0}(\mathcal{O}(1))$, which is an $(n+1)$-dimensional space with basis $z_{0}, \ldots, z_{n}$, the homogeneous coordinates on $\mathbb{P}_{\mathbb{C}}^{n}$. Applying (3.1), we have the following result.

Proposition 3.2. If $n$ is even, then $\mathbb{P}_{\mathbb{C}}^{n}$ has a unique real structure, the usual one, given by complex conjugation of the coordinates. If $n$ is odd, then $\mathbb{P}_{\mathbb{C}}^{n}$ has two possible real structures: the usual one, and another, which has no real points. In the second case, $\mathcal{O}(1)$ has a symplectic structure, and one can choose homogeneous coordinates $z_{0}, z_{1}, \ldots, z_{n}$ such that $\sigma$ is given by $\sigma\left(z_{0}, z_{1}, \ldots\right)=\left(-\bar{z}_{1}, \bar{z}_{0},-\bar{z}_{3}, \bar{z}_{2}, \ldots\right)$.

Note that if $\mathcal{O}(1)$ has a symplectic structure, then $\mathcal{O}(m)$ will have a real structure for $m$ even, and a symplectic structure for $m$ odd.

Now let us return to $\mathbb{P}_{\mathbb{C}}^{3}$ and the real structure $j$ on it, defined in $\S 1$, which we recognize as the unique nonstandard real structure on $\mathbb{P}_{\mathbb{C}}^{3}$. We will need to study the Grassmann variety $G(1,3)$ which parametrizes lines in $\mathbb{P}_{\mathbb{C}}^{3}$. Classically this is constructed as follows : a line $L \subseteq \mathbb{P}_{\mathbb{C}}^{3}$ corresponds to a 2-dimensional subspace of $\mathbb{C}_{\mathbb{C}}^{4}$. Let it be spanned by the vectors $\left(a_{0}, a_{1}, a_{2}, a_{3}\right)$ and $\left(b_{0}, b_{1}, b_{2}, b_{3}\right)$. Then consider the quantities $p_{i j}=a_{i} b_{j}-a_{j} b_{i}$ for $0 \leqq i<j \leqq 3$. These are the Plücker coordinates of the line $L$. They define a point in $\mathbb{P}_{\mathbb{C}}^{5}$ which depends only on $L$, not on the two vectors chosen. On the other hand, the quantities $p_{i j}$ satisfy the quadratic equation

$$
p_{01} p_{23}-p_{02} p_{13}+p_{03} p_{12}=0 \text {, }
$$

and one can show that the set of lines $\mathbb{P}_{\mathbb{C}}^{3}$ is in $1-1$ correspondence with the set of points in $\mathbb{P}_{\mathbb{C}}^{5}$ satisfying this equation. This then is the Grassmann variety $G(1,3)$, realized as a quadric hypersurface in $\mathbb{P}_{\mathbb{C}}^{5}$.

This Grassmann variety and the $\mathbb{P}_{\mathbb{C}}^{5}$ in which it is embedded are functorially associated with $\mathbb{P}_{\mathbb{C}}^{3}$. Therefore the real structure $j$ on $\mathbb{P}_{\mathbb{C}}^{3}$ induces a real structure on $G(1,3)$ and on $\mathbb{P}_{\mathbb{C}}^{5}$. Since the $p_{i j}$ are quadratic in the coordinates of $\mathbb{P}_{\mathbb{C}}^{3}$, we can expect that this $\mathbb{P}_{\mathbb{C}}^{5}$ will have the usual real structure. Indeed, this is easily verified directly. We know how $j$ acts on the coordinates of $\mathbb{P}_{\mathbb{C}}^{3}$. So we can compute the action of $j$ on $p_{01}=a_{0} b_{1}-a_{1} b_{0} \rightarrow \bar{a}_{1} \bar{b}_{0}+\bar{a}_{0} \bar{b}_{1}=\bar{p}_{01}$. Similarly

$$
\begin{array}{ll}
p_{01} \rightarrow \bar{p}_{01} & p_{13} \rightarrow \bar{p}_{02} \\
p_{02} \rightarrow \bar{p}_{13} & p_{12} \rightarrow-\bar{p}_{03} \\
p_{03} \rightarrow-\bar{p}_{12} & p_{23} \rightarrow \bar{p}_{23} .
\end{array}
$$

To see that this is the usual real structure on $\mathbb{P}_{\mathbb{C}}^{5}$, introduce new coordinates

$$
\begin{array}{lll}
x_{0}=p_{01}+p_{23} & x_{2}=p_{02}+p_{13} & x_{4}=p_{03}-p_{12} \\
x_{1}=p_{01}-p_{23} & x_{3}=i\left(p_{02}-p_{13}\right) & x_{5}=i\left(p_{03}+p_{12}\right) .
\end{array}
$$

Then $x_{i} \rightarrow \bar{x}_{i}$ for all $i$. 
In these new coordinates, the equation of $G(1,3)$ becomes

$$
x_{1}^{2}+x_{2}^{2}+x_{3}^{2}+x_{4}^{2}+x_{5}^{2}=x_{0}^{2} .
$$

Thus $G(1,3)$ inherits a real structure whose set of real points is a 4-sphere. We can identify this 4-sphere with the one in the map $\pi: \mathbb{P}_{\mathbb{C}}^{3} \rightarrow S^{4}$ of $\S 1$ in the following way. A real point of $G(1,3)$ is a point left fixed by the real structure $\sigma$ on $G(1,3)$. Thus it corresponds to a line in $\mathbb{P}_{\mathbb{C}}^{3}$ which is sent into itself by the map $j$. But these lines are precisely the fibres of the map $\pi$. We will call them the real lines in $\mathbb{P}_{\mathbb{C}}^{3}$ : they are those lines $L \subseteq \mathbb{P}_{\mathbb{C}}^{3}$ which have an induced real structure. That induced structure is of course the non-standard real structure on $\mathbb{P}_{\mathbb{C}}^{1}$, with no real points.

Now we are ready to apply these ideas to vector bundles and reinterpret conditions (3) and (4) of (1.1). For any rank 2 bundle $E$ on $\mathbb{P}_{\mathbb{C}}^{3}$, consider its restriction $\left.E\right|_{L}$ to a line $L \subseteq \mathbb{P}_{\mathbb{C}}^{3}$. It is known that any vector bundle on a line is a direct sum of line bundles. Therefore $\left.E\right|_{L} \cong \mathcal{O}(a) \oplus \mathcal{O}(b)$ for some integers $a, b$. If $c_{1}(E)=0$, then $a+b=0$. Furthermore, if $E$ is stable, a theorem of Grauert and Mülich [4] asserts that $a=b=0$ for almost all lines $L$, that is, for lines corresponding to an open dense subset of the Grassmann variety $G(1,3)$. A line for which $a, b \neq 0$ is called a jumping line of $E$. Barth [3] has shown that the set of jumping lines of a given bundle $E$ corresponds to a divisor $Z \subseteq G(1,3)$, i.e. a subvariety of codimension 1 in $G(1,3)$, and that $Z$ has degree $=c_{2}(E)$.

The condition (3) of (1.1) says that for every $p \in S^{4},\left.E\right|_{\pi^{-1}(p)}$ is the trivial bundle on $\pi^{-1}(p)$. In other words, $\pi^{-1}(p)$ is not a jumping line for $E$. Since the lines $\pi^{-1}(p)$ correspond exactly to the real points of $G(1,3)$, the condition (3) is equivalent to

(3') The divisor $Z$ of jumping lines of $E$ has no real points.

As for condition (4), we have already noted that the map $j$ would be a symplectic structure on $E$. Let us consider this condition in terms of the moduli space $M_{\mathbb{C}}\left(c_{2}\right)$. This moduli space is functorially associated with $\mathbb{P}_{\mathbb{C}}^{3}$, depending only on the integer $c_{2}$. Therefore it inherits a real structure $\sigma$. What are the real points of this real structure? If $E$ corresponds to a real point of $M_{\mathbb{C}}\left(c_{2}\right)$, that means $j^{*}(E) \cong E$ on $\mathbb{P}_{\mathbb{C}}^{3}$. Just as in the case of the sheaf $\mathcal{O}(1)$ discussed earlier, to give an isomorphism of $j^{*} E$ with $E$ is equivalent to giving a real or symplectic structure on $E$; furthermore, that structure is uniquely determined up to equivalence. Therefore we can rephrase condition (4) as follows:

$\left(4^{\prime}\right) E$ corresponds to a real point of $M_{\mathbb{C}}\left(c_{2}\right)$, and has a symplectic structure (not a real one).

Summing up, we can divide the problem (1.1) into four steps.

Step 1. Classify the stable rank 2 vector bundles on $\mathbb{P}_{\mathbb{C}}^{3}$ with $c_{1}=0, c_{2}>0$, and find their moduli space $M_{\mathbb{C}}\left(c_{2}\right)$.

Step 2. Identify the real structure on $M_{\mathbb{C}}\left(c_{2}\right)$ induced by $j$, and find its real points $M_{\mathbb{R}}\left(c_{2}\right)$.

Step 3. Among the real points $M_{\mathbb{R}}\left(c_{2}\right)$, find which ones correspond to bundles $E$ with a real structure, and which to bundles with a symplectic structure.

Step 4. Among those $E$ with a symplectic structure, find those whose divisor of jumping lines $Z$ has no real points. This will be the moduli space $M\left(c_{2}\right)$ of instantons, which thus appears as an open subset of $M_{\mathbb{R}}\left(c_{2}\right)$, defined by algebraic equalities and inequalities. 
Of course these steps may be very difficult to carry out. Note also that even if the complex moduli space $M_{\mathbb{C}}\left(c_{2}\right)$ is irreducible and nonsingular, for example, that tells us very little about $M\left(c_{2}\right)$, because the real points may fall into many connected components, and Step 4 may require removing some lower-dimensional subvarieties, and then taking certain connected components of the remainder.

We will carry out this program for $c_{2}=1,2$ in the next section.

\section{§4. The Moduli of Instantons with $c_{2}=1,2$}

First we treat the case $c_{2}=1$. If $E$ is a stable bundle with $c_{2}=1$, then its divisor of jumping lines $Z \leqq G(1,3)$ is a divisor of degree 1 . In other words, it is the intersection of $G(1,3)$ with a hyperplane $H$ in $\mathbb{P}_{\mathbb{C}}^{5}$. Now the quadric $G(1,3)$ determines a duality of the projective space $\mathbb{P}_{\mathbb{C}}^{5}$ with itself, whereby the hyperplane $H$ corresponds to a point $P_{E}$. Geometrically, $P_{E}$ is obtained as the common intersection of the tangent spaces to $G(1,3)$ at all the points of $Z=G(1,3) \cap H$ (see Fig. 1).

Fig. 1

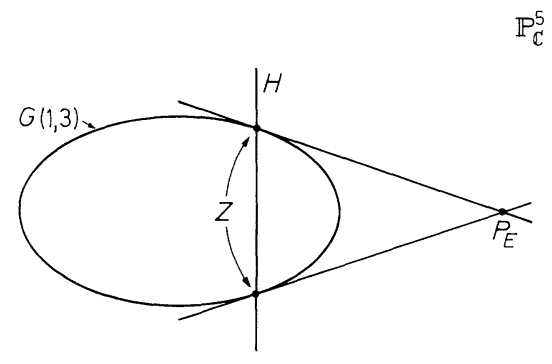

Theorem 4.1. $A$ stable bundle $E$ with $c_{1}=0$ and $c_{2}=1$ is completely determined by the point $P_{E}$ described above. Conversely, any point $P \in \mathbb{P}_{\mathbb{C}}^{5}$ not on $G(1,3)$ comes from a bundle. Therefore the moduli space $M_{\mathbb{C}}(1)=\mathbb{P}_{\mathbb{C}}^{5}-G(1,3)$.

This result is proved by Wever in his Berkeley thesis [10], also independently by Barth [3]. Atiyah et al. have a new differential-geometric proof of the corresponding statement in differential geometry, to appear in the expanded version of [1].

We have already discussed the induced real structure on $\mathbb{P}_{\mathbb{C}}^{5}$ and on $G(1,3)$. Thus we see that the real points of this space are $M_{\mathbb{R}}(1)=\mathbb{P}_{\mathbb{R}}^{5}-S^{4}$. This space has two connected components: the inside of $S^{4}$ and the outside of $S^{4}$. The points of $M_{\mathbb{R}}(1)$ correspond to stable bundles $E$ with a real or a symplectic structure. Since $\operatorname{dim}_{\mathbb{C}} H^{0}(E(1))=5$, the induced structure on $E(1)$ cannot be a symplectic structure (3.1). Therefore $E(1)$ has a real structure, which implies that $E$ must have had a symplectic structure. So both components of $\mathbb{P}_{\mathbb{R}}^{5}-S^{4}$ correspond to bundles with a symplectic structure.

It remains to determine which bundles $E$ have no real jumping lines. We can recover the divisor $Z$ of jumping lines by drawing all tangent lines from $P_{E}$ to 
$G(1,3)$ and taking their points of contact. If $P_{E}$ is a real point, the real jumping lines can be obtained by taking all real tangent lines from $P_{E}$ to $S^{4}$. So we see that if $P_{E}$ is outside $S^{4}$, there will be many real jumping lines; if $P_{E}$ is inside $S^{4}$ there will be none. So we have

Theorem 4.2. The moduli space $M(1)$ of instantons with $c_{2}=1$ is the inside of $S^{4}$ in $\mathbb{R}^{5}$. In particular, it is a contractible real open 5-manifold.

We make two further remarks about this moduli space. We have already seen for $c_{2}=1$ that $H^{0}(E(1)) \neq 0$, and that any stable $E$ with $c_{2}=1$ corresponds to a union of two lines in $\mathbb{P}_{\mathbb{C}}^{3}$. The space $H^{0}(E(1))$ corresponds to the lines through the point $P_{E}$. Such a line meets $G(1,3)$ in two points, which correspond to the two lines in $\mathbb{P}_{\mathbb{C}}^{3}$ determined by the given section $s \in H^{0}(E(1))$. So we see that the possible pairs of lines which occur for a given bundle $E$ correspond to pairs of points on $G(1,3)$ which are collinear with $P_{E}$. In particular, if $P \in M(1)$, there always are pairs of points on $S^{4}$ collinear with $P$, so the bundle $E$ can be constructed from two points of $S^{4}$. The scalars $\lambda_{0}, \lambda_{1}$ depend on the position of $P$ along the line joining those two points; $\lambda_{0}, \lambda_{1}>0$ since $P$ is inside $S^{4}$. Therefore we have

Corollary 4.3. The only possible instantons with $c_{2}=1$ are those already constructed by the physicists using two points $p_{0}, p_{1} \in S^{4}$ and real scalars $\lambda_{0}, \lambda_{1}>0$.

Our other remark concerns the action of the group PGL(3) of automorphisms of $\mathbb{P}_{\mathbb{C}}^{3}$. This group acts transitively on $M_{\mathbb{C}}(1)$, so we see that any two stable bundles with $c_{2}=1$ are equivalent by an automorphism of $\mathbb{P}_{\mathbb{C}}^{3}$. Since PGL(3) depends functorially on $\mathbb{P}_{\mathbb{C}}^{3}$, it inherits a real structure from the real structure $j$. The group of real points of PGL(3) for this real structure is just the connected component of the identity in $\mathrm{SO}(5,1)$. It acts transitively on the inside of $S^{4}$, so we see that the space of instantons $M(1)$ is a homogeneous space under this group.

Now we come to the case $c_{2}=2$. Our main result is this.

Theorem 4.4. The moduli space $M(2)$ of instantons with $c_{2}=2$ is a connected, but not simply connected, real 13-dimensional manifold. Every instanton with $c_{2}=2$ can be obtained by the physicists' construction starting from 3 points $p_{0}, p_{1}, p_{2} \in S^{4}$ and three real numbers $\lambda_{0}, \lambda_{1}, \lambda_{2}>0$.

Step 1 . We start by describing the moduli space $M_{\mathbb{C}}(2)$ of stable bundles on $\mathbb{P}_{\mathbb{C}}^{3}$ with $c_{1}=0$ and $c_{2}=2$. We have already seen that for any such bundle $E$, $H^{0}(E(1)) \neq 0$, so that $E$ corresponds to a curve $Y$ of degree 3 with $\omega_{Y} \cong \mathcal{O}_{Y}(-2)$. One can show that the degenerate cases of $Y$ give no new bundles, so we may assume that $Y$ is three skew lines. A theorem of classical projective geometry asserts that a set of three skew lines in $\mathbb{P}_{\mathbb{C}}^{3}$ lies on a unique nonsingular quadric surface $Q$. This quadric $Q$ can be constructed as the union of all other lines which meet each of the three given lines. Furthermore, one knows that a nonsingular quadric $Q$ in $\mathbb{P}_{\mathbb{C}}^{3}$ is isomorphic to $\mathbb{P}_{\mathbb{C}}^{1} \times \mathbb{P}_{\mathbb{C}}^{1}$. The points of the two factors correspond to the lines on the quadric surface.

Of course the curve $Y$ depends on the choice of a section $s \in H^{0}(E(1))$. If $s^{\prime} \in H^{0}(E(1))$ is another section, then we get another curve $Y^{\prime}$ which is a union of three lines, but one can show that $Y^{\prime}$ determines the same quadric surface $Q$. Thus 
$Q$ depends only on $E$; as $s \in H^{0}(E(1))$ varies, the curves $Y$ move in a linear system on $Q$ of type $(3,0)$, meaning that each divisor $Y$ consists of three lines in one of the two families of lines on $Q$. This picks out for us one of the two factors $\mathbb{P}_{\mathbb{C}}^{1}$ of $Q$, and the linear system of curves $Y$ then corresponds to a linear system $g_{3}^{1}$ of degree 3 and dimension 1 on $\mathbb{P}_{\mathbb{C}}^{1}$. Degree 3 means sets of 3 points moving; dimension 1 means it is a 1-parameter family-this is because $\operatorname{dim} H^{0}(E(1))=2$. Furthermore, one can show that this $g_{3}^{1}$ has no base points, that is, all three points are effectively moving.

Theorem 4.5. A stable bundle $E$ on $\mathbb{P}_{\mathbb{C}}^{3}$ with $c_{1}=0, c_{2}=2$ determines

(a) a nonsingular quadric surface $Q$

(b) a choice of one of the two factors $Q=\mathbb{P}_{\mathbb{C}}^{1} \times \mathbb{P}_{\mathbb{C}}^{1}$, and

(c) a linear system $g_{3}^{1}$ without base points on $\mathbb{P}_{\mathbb{C}}^{1}$.

Conversely, any set of such data (a), (b), (c) comes from a unique bundle E.

This enables us to describe the moduli space $M_{\mathbb{C}}(2)$ as a fibre space. The quadric surfaces in $\mathbb{P}_{\mathbb{C}}^{3}$ are parametrized by $\mathbb{P}_{\mathbb{C}}^{9}$. Let $\Delta \cong \mathbb{P}_{\mathbb{C}}^{9}$ be the subset corresponding to the singular or degenerate quadric surfaces. Then $M_{\mathbb{C}}(2)$ is fibred over $\mathbb{P}_{\mathbb{C}}^{9}-\Delta$.

$$
\begin{gathered}
M_{\mathbb{C}}(2) \\
\downarrow U \Perp U \\
\mathbb{P}_{\mathbb{C}}^{9}-\Delta
\end{gathered}
$$

The fibre is the disjoint union of two copies of the variety $U$ which parametrizes the set of possible $g_{3}^{1}$ with no base points on $\mathbb{P}_{\mathbb{C}}^{1}$. A $g_{3}^{1}$ is determined by a 2-dimensional subspace of the 4-dimensional vector space $H^{0}\left(\mathbb{P}_{\mathbb{C}}^{1}, \mathcal{O}(3)\right)$. Thus the set of all possible $g_{3}^{1}$ is parametrized by the Grassmann variety $G(1,3)$; those with no base points form an open subset $U$. Note that even though the fibre of this fibration is disconnected, the whole space $M_{\mathbb{C}}(2)$ is connected, because by moving 3 lines in $\mathbb{P}_{\mathbb{C}}^{3}$, one can get from one factor of $Q$ to the other.

So we see that $M_{\mathbb{C}}(2)$ is an irreducible nonsingular variety of dimension 13. Step 2. Next we must determine the real structure on $M_{\mathbb{C}}(2)$ and find its real points. We do this by using the fibration just described.

Since $\mathbb{P}_{\mathbb{C}}^{9}$ parametrizes quadrics in $\mathbb{P}_{\mathbb{C}}^{3}$, it is the projective space associated to the vector space $H^{0}\left(\mathcal{O}_{\mathbb{P}^{3}}(2)\right)$. Since $\mathcal{O}_{\mathbb{P}^{3}}(1)$ has a symplectic structure, $\mathcal{O}_{\mathbb{P} 3}(2)$ will have a real structure. Therefore the induced real structure on $\mathbb{P}_{\mathbb{C}}^{9}$ is the standard one, and its real points are $\mathbb{P}_{\mathbb{R}}^{9}$.

Next, what are the real points of $\Delta$ ? They correspond to singular or degenerate quadric surfaces in $\mathbb{P}_{\mathbb{C}}^{3}$, which can have no real points, since $\mathbb{P}_{\mathbb{C}}^{3}$ has no real points. This rules out a quadric cone (the vertex would have to be a real point) and a doubled plane (that plane would have to be real, but $\mathbb{P}_{\mathbb{C}}^{3}$ has no real planes). The only possibility is the union of a plane with its conjugate plane. Since the planes in $\mathbb{P}_{\mathbb{C}}^{3}$ are parametrized by another $\mathbb{P}_{\mathbb{C}}^{3}$, with the same real structure $j$, we see that $\Delta_{\mathbb{R}}$ is isomorphic to the quotient space $\mathbb{P}_{\mathbb{C}}^{3} / j$, which is a compact real 6-manifold.

Notice that $\mathbb{P}_{\mathbb{R}}^{9}-\Delta_{\mathbb{R}}$ is connected (since $\Delta_{\mathbb{R}}$ has codimension $>1$ ) but not simply connected (because $\mathbb{P}_{\mathbb{R}}^{9}$ is not simply connected).

To study the fibre $U \Perp U$, fix a point in the base $\mathbb{P}_{\mathbb{R}}^{9}-\Delta_{\mathbb{R}}$. This corresponds to a nonsingular quadric $Q$ with a real structure $\sigma$. We know that $Q \cong \mathbb{P}_{\mathbb{C}}^{1} \times \mathbb{P}_{\mathbb{C}}^{1}$ over $\mathbb{C}$. 
The real structure $\sigma$ cannot interchange the two factors, because then the intersection of a line with its conjugate line would be a real point. Therefore $\sigma$ leaves each factor fixed, so each factor $\mathbb{P}_{\mathbb{C}}^{1}$ has a real structure. The sheaf $\mathcal{O}_{Q}(1)$ on $Q$ is induced from $\mathcal{O}_{\mathbb{P}^{3}}(1)$. Therefore it has a symplectic structure. On the other hand,

$$
\mathcal{O}_{Q}(1) \cong p_{1}^{*} \mathcal{O}_{\mathbb{P}^{1}}(1) \otimes p_{2}^{*} \mathcal{O}_{\mathbb{P}^{1}}(1),
$$

where $p_{1}$ and $p_{2}$ are the two projections. Therefore $\mathcal{O}_{\mathbb{P} 1}(1)$ must have a real structure on one factor, and a symplectic structure on the other. Thus one factor $\mathbb{P}^{1}$ has the standard real structure, and the other has the nonstandard structure. Correspondingly, the $G(1,3)$ of which $U$ is an open subset has the standard real structure in one case, and the nonstandard one, described above, in the other case. Step 3. It is not hard to see that the bundles $E$ with a symplectic structure correspond to the choice of factor $\mathbb{P}_{\mathbb{C}}^{1}$ with the standard real structure; those $E$ with a real structure correspond to the other factor. So we can ignore one $U$ in the fibre. The one we need is an open subset of a $G(1,3)$ with its standard real structure.

Step 4. The analysis of the divisor of jumping lines and the determination of which bundles have no real jumping lines is the most delicate part of the proof.

First we show how to describe the divisor $Z$ of jumping lines of a bundle $E$. As before, $E$ determines a quadric $Q \subseteq \mathbb{P}_{\mathbb{C}}^{3}$, and a choice of factor $\mathbb{P}_{\mathbb{C}}^{1}$ of $Q$. The points of $\mathbb{P}_{\mathbb{C}}^{1}$ correspond to lines in $Q$, which in turn correspond to points of the Grassmann variety $G(1,3)$ of lines in $\mathbb{P}_{\mathbb{C}}^{3}$. As $p \in \mathbb{P}_{\mathbb{C}}^{1}$ varies, its image in $G(1,3)$ describes a conic $\gamma \subseteq G(1,3)$. Let $\pi$ be the unique plane of $\mathbb{P}_{\mathbb{C}}^{5}$ containing $\gamma$. Now on $\gamma$ we have the $g_{3}^{1}$ coming from $E$. For each divisor $P_{1}+P_{2}+P_{3}$ in the $g_{3}^{1}$, draw the tangent lines to $\gamma$ at $P_{1}, P_{2}, P_{3}$, and let them intersect at $X_{1}, X_{2}, X_{3}$. Then as $P_{1}+P_{2}+P_{3}$ varies in $g_{3}^{1}$, the points $X_{1}, X_{2}, X_{3}$ move on another conic $\Gamma$ (see Fig. 2).

Fig. 2

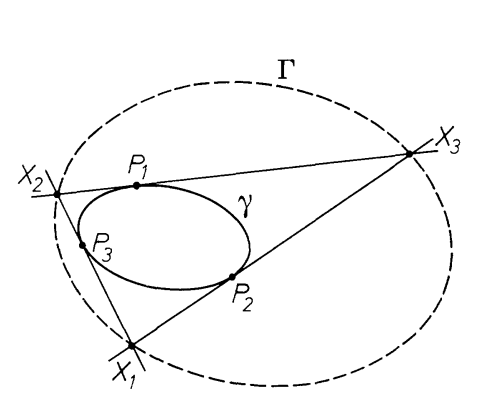

Now let $\pi^{*} \subseteq \mathbb{P}_{\mathbb{C}}^{5}$ be the dual plane to $\pi$ under the duality determined by the quadric $G(1,3)$. Let $W$ be the cone over the conic $\Gamma$ with vertex $\pi^{*}$. Then $W$ is a quadric hypersurface in $\mathbb{P}_{\mathfrak{C}}^{5}$, and the divisor $Z$ of jumping lines of $E$ is just $W \cap G(1,3)$.

The question whether $Z$ has any real points is equivalent, by projection into the plane $\pi$, to the question whether the conic $\Gamma$ meets the inside of the conic $\gamma$. This depends of course on the $g_{3}^{1}$. One can show that there is just one connected 
component of $U_{\mathbb{R}}$ for which the corresponding $\Gamma$ does not meet the inside of $\gamma$. In terms of three real points on $\gamma$, this corresponds to specifying three positive real numbers $\lambda_{0}, \lambda_{1}, \lambda_{2}$. Thus these solutions are the same as the ones found by the physicists.

We conclude that the space of instantons $M(2)$ is fibred over $\mathbb{P}_{\mathbb{R}}^{9}-\Delta_{\mathbb{R}}$, with fibre an open connected real 4-manifold. Therefore $M(2)$ itself is connected but not simply connected, since the same is true of the base. This completes the proof of (4.4).

A couple of further remarks. One can also show in this case that the bundle $E$ is uniquely determined by its divisor of jumping lines $Z$. It is not known whether this is true for stable bundles with arbitrary $c_{2}$.

The group PGL(3) of automorphisms of $\mathbb{P}_{\mathbb{C}}^{3}$ acts on $M_{\mathbb{C}}(2)$, but it is not transitive. There is a 1-parameter family of inequivalent bundles under this action. I am not sure how to distinguish them, but a good guess seems to be the following: a $g_{3}^{1}$ without base points determines a degree 3 map of $\mathbb{P}_{\mathbb{C}}^{1} \rightarrow \mathbb{P}_{\mathbb{C}}^{1}$. This map will have 4 branch points in $\mathbb{P}_{\mathbb{C}}^{1}$. The cross ratio of these four points (modulo action of the symmetric group on 4 letters) will give an invariant of the bundle $E$, similar to the $j$-invariant of an elliptic curve. Perhaps this invariant characterizes the equivalence classes under the action of PGL(3).

Acknowledgements. I would like to thank M. F. Atiyah and I. M. Singer for pointing out that the work I was doing on stable vector bundles was related to physics, and for explaining the connection between the two subjects. I would also like to thank G. Lugo for taking notes of the lectures on which this article is based.

\section{References}

1. Atiyah,M.F., Hitchin, N.J., Singer,I.M.: Deformations of instantons. Proc. Nat. Acad. Sci. USA 74, 2662-2663 (1977)

2. Atiyah, M.F., Ward, R. S.: Instantons and algebraic geometry. Commun. math. Phys. 55, 117-124 (1977)

3. Barth, W.: Some properties of stable rank-2 vector bundles on $\mathbb{P}_{n}$. Math. Ann. 226, 125-150 (1977)

4. Grauert,H., Mülich,G.: Vektorbündel vom Rang 2 über dem n-dimensionalen komplexprojektiven Raum. manuscripta math. 16, 75-100 (1975)

5. Hartshorne, R.: Algebraic geometry. In: Graduate texts in mathematics, Vol. 52. Berlin-HeidelbergNew York: Springer 1977

6. Hartshorne, R.: Moduli of stable vector bundles on $\mathbb{P}^{3}$. In preparation

7. Jackiw, R., Nohl,C., Rebbi,C.: Conformal properties of pseudoparticle configurations. Phys. Rev. D 15, 1642-1646 (1977)

8. Maruyama, M. : Moduli of stable sheaves. I. J. Math. Kyoto Univ. 17, 91-126 (1977)

9. Serre,J.-P., Géométrie algébrique et géométrie analytique. Ann. Inst. Fourier 6, 1-42 (1956)

10. Wever, G.: Ph. D.Thesis, Berkeley (1977)

Communicated by J. Glimm

Received November 28, 1977

\section{Note Added in Proof}

Recently Atiyah and Hitchin in Oxford and Drinfeld and Manin in Moscow have found a linear algebra construction, based on earlier work of Horrocks and Barth, which gives all stable vector bundles coming from instantons. 
